\title{
Estimation of Multichannel Magnetometer Noise Floor in Ordinary Laboratory Conditions
}

\author{
D. PrasličKa, P. LipovskÝ*, J. HudÁK And M. ŠMelko
}

Faculty of Aeronautics, Technical University of Košice, Rampová 7, 04121 Košice, Slovakia

\begin{abstract}
The article deals with the method of the inherent noise estimation in each channel of a four channel relax-type magnetometer in general laboratory conditions. The day-long development of ambient magnetic field has been recorded and then the data were processed by correlation methods to separate inherent noises of the channels from ambient noise in the statistically best times. The method is applicable for noise estimation of any multi-channel sensoric system and also for the separation of deterministic and stochastic components of signals
\end{abstract}

DOI: 10.12693/APhysPolA.131.1123

PACS/topics: 89.20.Kk, 07.55.--w, 75.50.Kj

\section{Introduction}

As at the department we do design of magnetometers for scientific and industrial applications, the serious estimation of their inherent noises is among other parameters one of the crucial tasks [1]. Especially, when the sensors have open magnetic circuit and they use the relaxation working principle $[2,3]$. However, precise magnetic measurements of this type are affected by ambient magnetic noise that is often non-stationary and spatially heterogeneous. Often there is not the possibility to perform the measurements in a magnetically shielded chamber, so the magnetic parameters have to be estimated from large amounts of data with the help of statistical processing.

\section{Theory}

Suppose that the output signal of the sensor is a linear superposition of the ambient input signal that is the sum of deterministic and stochastic components and the inherent noise of the sensor. The variance of the output signal is

$$
\operatorname{var}(x)=\frac{1}{N-1} \sum_{i=1}^{N}\left(x_{i}-\bar{x}\right)\left(x_{i}-\bar{x}\right)
$$

which represents the total power of the signal. The covariance of two output signals

$$
\operatorname{cov}(x, y)=\frac{1}{N-1} \sum_{i=1}^{N}\left(x_{i}-\bar{x}\right)\left(y_{i}-\bar{y}\right)
$$

is the power of ambient input signal only, assuming that the inherent noises of two different sensor channels are uncorrelated [4]. The covariance matrix for four channels is defined as:

$$
\left(\begin{array}{cccc}
\operatorname{var}\left(x_{1}\right) & \operatorname{cov}\left(x_{1}, x_{2}\right) & \operatorname{cov}\left(x_{1}, x_{3}\right) & \operatorname{cov}\left(x_{1}, x_{4}\right) \\
\operatorname{cov}\left(x_{2}, x_{1}\right) & \operatorname{var}\left(x_{2}\right) & \operatorname{cov}\left(x_{2}, x_{3}\right) & \operatorname{cov}\left(x_{2}, x_{4}\right) \\
\operatorname{cov}\left(x_{3}, x_{1}\right) & \operatorname{cov}\left(x_{3}, x_{2}\right) & \operatorname{var}\left(x_{3}\right) & \operatorname{cov}\left(x_{3}, x_{4}\right) \\
\operatorname{cov}\left(x_{4}, x_{1}\right) & \operatorname{cov}\left(x_{4}, x_{2}\right) & \operatorname{cov}\left(x_{4}, x_{3}\right) & \operatorname{var}\left(x_{4}\right)
\end{array}\right),
$$

\footnotetext{
*corresponding author; e-mail: pavol.lipovsky@tuke.sk
}

where $x_{1}, x_{2}, x_{3}$ and $x_{4}$ are appropriate output signals of four channel magnetometer sensors located in parallel and spatially placed close to each other. This total power matrix has the value of the total power including inherent noise of each channel in the diagonal and off the diagonal there are common powers of the signals between the corresponding channels. Assuming homogeneous and stationary magnetic field as the input signal, the off-diagonal elements would be equal. The standard deviation of the inherent noise of the $i$-th channel could be then calculated as

$$
\sigma_{i}=\sqrt{\operatorname{var}\left(x_{i}\right)-\operatorname{cov}\left(x_{i}, x_{j}\right)} .
$$

Unfortunately, aforementioned assumption is not fulfilled in general laboratory conditions. Spectral analysis clearly shows that the dominant component of the heterogeneity and non-stationarity of the field in low frequency range are interferences that occur mostly (and almost everywhere) on the industrial frequencies of $50 \mathrm{~Hz}$ and $150 \mathrm{~Hz}$, sometimes also on higher harmonics. With the help of the autocorrelation and cross-correlation we can evaluate the powers of these deterministic interferences. By the subtraction of these powers from the appropriate total power matrix elements we remove the spatial heterogeneity of the interferences. Consequently, only the random elements remain in the resulting noise power matrix. However, the magnetic field have to be stationary during the measurement. So, we have to choose the appropriate statistic criteria and select the most suitable time intervals of the measurements.

\section{Measurements and results}

In this article, as the multichannel sensoric system the VEMA series four channel magnetometer with simultaneous sampling [5] developed at our department was used. The magnetometer channels were calibrated using the algorithms based on the neural networks [6]. The bandwidth of the magnetometer is $250 \mathrm{~Hz}(-3 \mathrm{~dB})$, the sampling frequency $f_{s}=1000 \mathrm{~Hz}$ and the full scale range is $\pm 70 \mu \mathrm{T}$. Considering the statements from the theory, we had to evaluate the stationarity of the ambient (measured) magnetic field at first. A 24 hour time develop- 
ment of the AC magnetic field recorded from Saturday May 21st 00:00 a.m. is shown in Fig. 1 for the four magnetometer channels $-x_{1}, x_{2}, x_{3}, x_{4}$. As it can be seen, there are obvious disturbances in the time development of the standard deviations as a result of the power grid activity in the laboratory or near the laboratory. We can also estimate the best time interval for the measurements, but this is not necessary, because we are processing large amount of the data and in the utility program written in the $\mathrm{C}$ language rules to avoid processing of data files with non-stationary signals are implemented, based on the differences of the cross-correlations symmetrically along the diagonal of the matrix, and a rule to detect spatially heterogeneous signals, based on the differences among the off-diagonal elements.

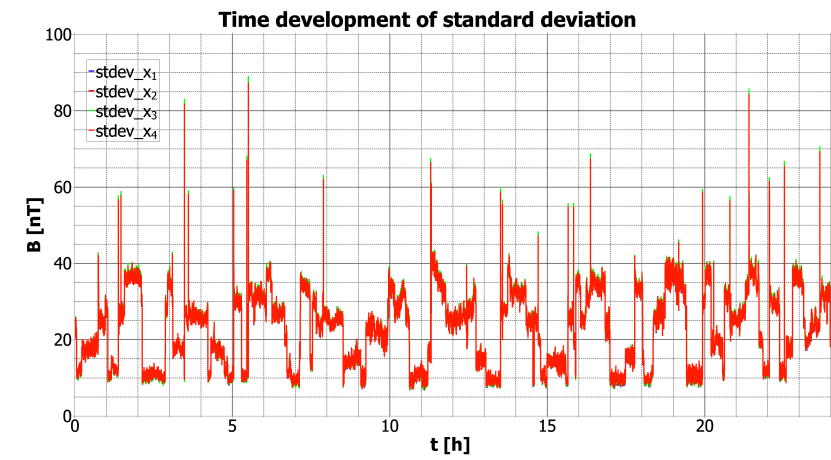

Fig. 1. 24 hour development of the magnetic field standard deviation at the measurement place.

For the purpose of the measurements, a fixture made from hard wood was made on a CNC machine, to suppress the uncertainties in the positioning of the sensors. All of the sensors were placed in the fixture in parallel in the points of a square with the side of $100 \mathrm{~mm}$

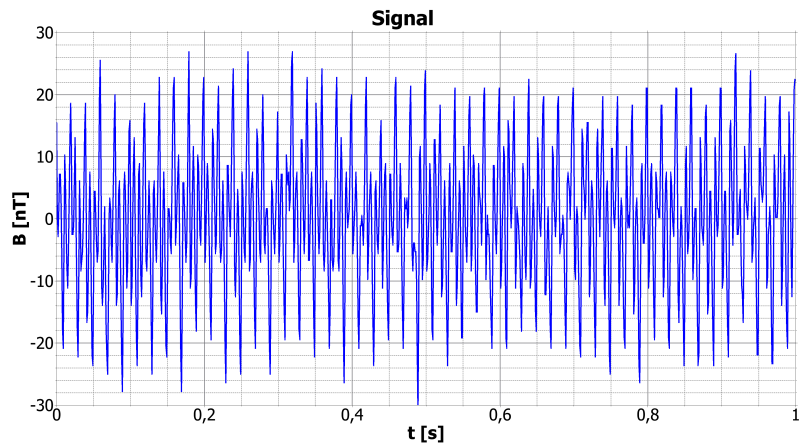

Fig. 2. Time development of a typical recorded signal.

Figures 2-5 show typical measured signal in the measurement place, with the subtracted mean value for a better illustration. From the figures we can assume that the dominant interferences are on the industrial frequency and its harmonics.

Prior to the data recording, we had to find a place in our laboratory, where the ambient DC field is homogeneous within tolerable limits - to evaluate the sensors in similar working points on their transfer characteristics, and where also the interference has acceptable values. The data were recorded in $5 \mathrm{~s}$ time intervals in $2 \mathrm{~s}$ long recordings.

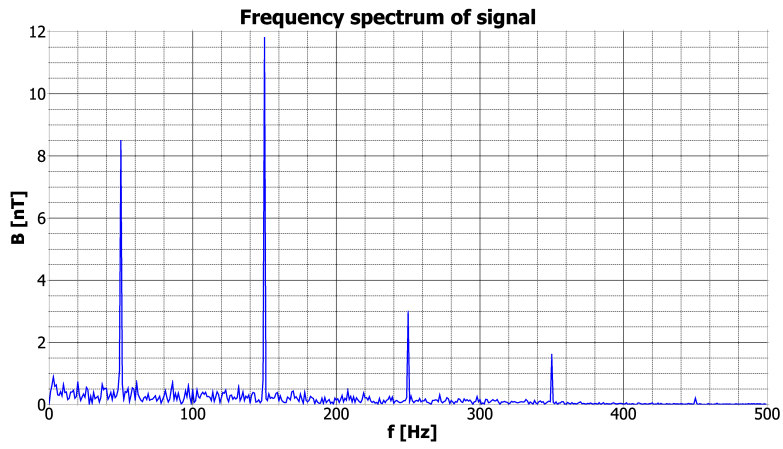

Fig. 3. Frequency spectrum of the typical recorded signal.

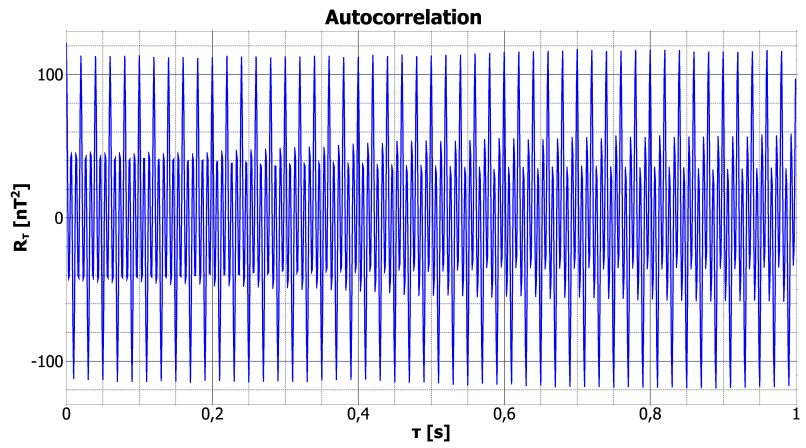

Fig. 4. Autocorrelation of the typical recorded signal.

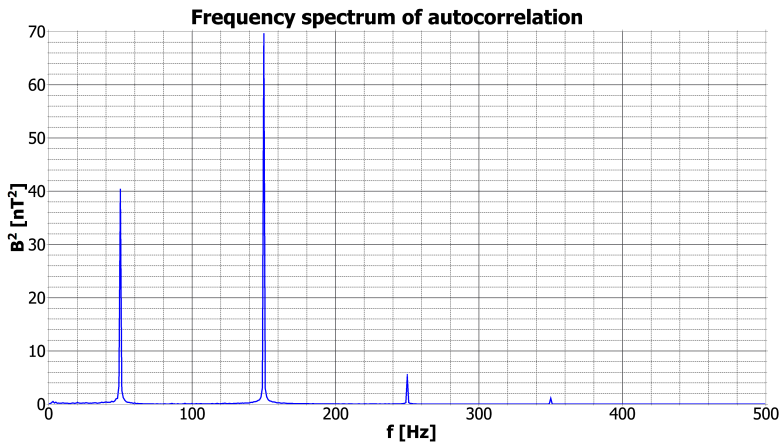

Fig. 5. Power frequency spectrum of the typical recorded signal.

After $24 \mathrm{~h}$ of measurement, each of the data recordings was processed in these steps:

- the covariance matrix was computed - this is the total power matrix,

- the deterministic power matrix was computed with the help of the cross-correlation $-1 \mathrm{~s}$ of the channel with number equal to the first index was correlated 
with $2 \mathrm{~s}$ of the of the channel with the number equal to the second index, then with the discrete Fourier transformation the powers of industrial interferences were computed,

- the noise matrix was computed as the difference between the total power matrix and deterministic power matrix elements,

- the stationarity and homogeneity rules were applied - based on these the recording was or wasn't excluded from inclusion into statistics.

The matrices were averaged to obtain the average noise matrix. For the stationarity tolerance of $1 \mathrm{nT}^{2}$ and homogeneity tolerance of $2 \mathrm{nT}^{2}$, we obtained the average total power matrix:

$$
\left(\begin{array}{cccc}
101.124 & 92.092 & 91.958 & 91.449 \\
92.092 & 102.814 & 92.651 & 91.129 \\
91.958 & 92.651 & 102.384 & 91.878 \\
91.449 & 92.129 & 91.878 & 109.252
\end{array}\right)
$$

the average deterministic power matrix:

$$
\left(\begin{array}{llll}
85.650 & 86.404 & 86.232 & 85.715 \\
86.336 & 87.232 & 87.006 & 86.497 \\
86.186 & 87.012 & 86.908 & 86.361 \\
85.662 & 86.505 & 86.343 & 86.063
\end{array}\right),
$$

and the average noise matrix:

$$
\left(\begin{array}{cccc}
15.473 & 5.690 & 5.726 & 5.734 \\
5.756 & 15.583 & 5.645 & 5.632 \\
5.773 & 5.639 & 15.476 & 5.519 \\
5.785 & 5.623 & 5.535 & 23.189
\end{array}\right),
$$

where on the diagonal we have average noise estimations for the four channels, $x_{1}, x_{2}, x_{3}, x_{4}$. Off-diagonal elements represent the ambient random interferences and different values point to the heterogeneity of the field. As visible from almost equality of the average noise matrix off-diagonal elements, the homogeneity and stationarity of the field was acceptable.

We tested this method in different places and the results were always almost the same, showing that the 4-th, the $x_{4}$-channel has the worst properties - combination of the sensor and electronics noise. Finally, to obtain the noise floor estimation, we can subtract the minimum value of the off-diagonal elements from the diagonal values - Table 1, but we have to add the quantization noise, in our case $0.182 \mathrm{nT}^{2}$.

Noise estimations.

TABLE I

\begin{tabular}{c|c|c|c|c}
\hline \hline channel & 1 & 2 & 3 & 4 \\
\hline noise power $\left[\mathrm{nT}^{2}\right]$ & 10.138 & 10.247 & 10.141 & 17.853 \\
$\sigma[\mathrm{nT}]$ & 3.184 & 3.201 & 3.185 & 4.225
\end{tabular}

Based on the theory and previous measurements of the frequency characteristics of the magnetometer, the bandwidth and based on the equation:

$$
P S D_{i}=\frac{\sigma_{i}^{2}}{250}
$$

we can compute the power spectral density (PSD) and linear spectral density (LSD) estimations - Table II, in $1-250 \mathrm{~Hz}$ bandwidth.

Noise estimations.

TABLE II

\begin{tabular}{c|c|c|c|c}
\hline \hline channel & 1 & 2 & 3 & 4 \\
\hline $\mathrm{PSD}\left[\mathrm{nT}^{2} / \mathrm{Hz}\right]$ & 0.0405 & 0.0410 & 0.0406 & 0.0714 \\
$\mathrm{LSD}\left[\mathrm{nT} / \mathrm{Hz}^{1 / 2}\right]$ & 0.201 & 0.203 & 0.201 & 0.267
\end{tabular}

\section{Conclusions}

The method used to estimate the noise of multichannel sensoric system discussed in this paper can be used in general laboratory conditions. Although there are some rules to follow, this method is relatively simple and is based on the statistical data processing and spectral analysis. This method can be used to estimate noise also for different types of sensors, also for novel magnetic microwire sensors in biomedical applications [7].

The matrices obtained with this method contain information not only about the noise of each channel of the multichannel system but also about ambient field - the diagonal values of the power matrix for the deterministic field and the off-diagonal elements of the noise matrix for the random fields.

\section{Acknowledgments}

This work has been supported by the grant agencies of the Slovak Republic grants VEGA 1/0201/16 and APVV0266-10.

\section{References}

[1] P. Ripka, Sensors Actuat. A Phys. 106, 8 (2003).

[2] D. Praslicka, IEEE Trans. Magn. 30, 934 (1994).

[3] P. Butvin, D. Praslicka, J. Blazek, B. Butvinova, Sensors Actuat. A Phys. 106, 22 (2003).

[4] D. Praslicka, M. Smelko, J. Blazek, J. Hudak, P. Lipovsky, N. Flachbart, Acta Phys. Pol. A 126, 86 (2014).

[5] J. Hudak, J. Blazek, D. Praslicka, I. Mikita, P. Lipovsky, P. Gonda, J. Electr. Eng. 61, 28 (2010).

[6] T. Kliment, D. Praslicka, K. Draganova, J. Blazek, J. Electr. Eng. 66, 157 (2015).

[7] R. Hudak, R. Varga, I. Polacek, P. Klein, I. Skorvanek, V. Komanicky, R. Perez Del Real, M. Vazquez, Phys. Status Solidi A 213, 377 (2016). 